\title{
Continuous catalytic depolymerisation and conversion of industrial kraft lignin into low-molecular-weight aromatics
}

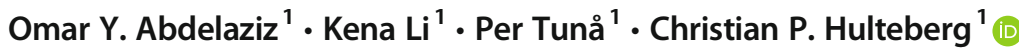 \\ Received: 13 September 2017 / Revised: 14 September 2017 / Accepted: 15 November 2017 / Published online: 27 November 2017 \\ (C) The Author(s) 2017. This article is an open access publication
}

\begin{abstract}
Base-catalysed depolymerisation of lignin using sodium hydroxide has been shown to be an effective approach towards exploiting industrial (technical) lignins within the pulp and paper industry. In the present work, a pine kraft lignin (Indulin AT) which is precipitated from black liquor of linerboard-grade pulp was depolymerised via base catalysis to produce lowmolecular-mass aromatics without any organic solvent/capping agent in a continuous-flow reactor setup for the first time. The catalytic conversion of lignin was performed/screened at temperatures varying from 170 to $250^{\circ} \mathrm{C}$, using $\mathrm{NaOH} /$ lignin weight ratio $\approx 1$ with $5 \mathrm{wt} \%$ lignin solids loadings for residence times of 1,2 and $4 \mathrm{~min}$, respectively, with comprehensive characterisation of substrate and produced reaction mixtures. The products were characterised using size exclusion chromatography (SEC), nuclear magnetic resonance spectroscopy (NMR) and supercritical fluid chromatography-diode array detector-tandem mass spectrometry (SFC-MS). The optimum operating conditions for such depolymerisation appeared to be at $240{ }^{\circ} \mathrm{C}$ and $30 \mathrm{~h}^{-1}$, yielding the highest concentration of low-molecular-weight phenolics below the coking point. It was also found that the depolymerised lignin products exhibited better chemical stability during long-term storage at lower temperatures $\left(\sim 4{ }^{\circ} \mathrm{C}\right)$.
\end{abstract}

Keywords Biorefineries $\cdot$ Lignin valorisation $\cdot$ Lignin depolymerisation $\cdot$ Biomass conversion $\cdot$ Continuous-flow reactor system $\cdot$ Renewable chemicals

\section{Introduction}

Lignin, a complex and water-insoluble aromatic biopolymer, is attracting much attention owing to its potential as a renewable resource for the production of value-added chemicals, fuels, aromatics and bio-based materials. Conventionally, most large-scale industrial plants that utilise plant polysaccharides have burned lignin to generate the heat and power needed for biomass transformation and/or product drying. The emergence of biorefineries that convert cellulosic biomass into liquid biofuels will generate considerably more lignin than necessary to power the operation in addition to the lignin produced/combusted by the pulp and paper industry, and accordingly, efforts are underway to convert it into more valuable products [1]. It should be pointed out that lignin is the

Christian P. Hulteberg

Christian.Hulteberg@chemeng.lth.se

1 Department of Chemical Engineering, Lund University, P.O. Box 124, SE-221 00 Lund, Sweden sole renewable available feedstock in nature that constitutes typical aromatic rings. Recent figures suggest that more than 130 million tons of lignin are presently liberated only from the pulp and paper industry, most of which is directly used on-site $[2,3]$. Hence, the production of aromatic chemicals from lignin - the conversion process - is considered to be highly atom-economic; and the reason is that most of the carbon, hydrogen and oxygen atoms are well-reserved within the products. Designing new innovative processes/technologies to produce high-value products and subsequent scaling up of these technologies to produce such lignin-derived products at the commercial scale level and on a life cycle basis is a critical goal of many researchers currently working in the field of lignin utilisation $[4,5]$.

The challenges in employing lignin as an initial raw material for low-molecular-weight chemicals originate from the fact that the biopolymer is markedly heterogeneous - in many different aspects [3]. Firstly, lignin is heterogeneous in the sense that various plants build their lignin structures with different proportions of the constitutive building blocks. Basically, its streams differ in structure depending majorly 
on the method of isolation and source of plant [6]. Secondly, the cross-linking patterns are substantially stochastically created, and the lignin macromolecule is connected to the hemicellulose within the plant as well. There is therefore heterogeneity for lignin even from the same plant, highlighting the challenges towards finding efficient lignin valorisation strategies. Lignin can be specifically outlined as an amorphous biopolymer that is composed of three primary aromatic unitsphenylpropane units - and termed as monolignols, namely $p$ coumaryl, coniferyl and sinapyl alcohols (Fig. 1). These units are linked together randomly by different types of ether/aryl ether $(\mathrm{C}-\mathrm{O})$ and carbon-carbon $(\mathrm{C}-\mathrm{C})$ bonds $[7,8]$, where the aryl ether linkages are spotted to be more easily cleaved [9] in comparison to the stable $\mathrm{C}-\mathrm{C}$ linkages that are considered to be much more resistant towards chemical depolymerisation.

Depolymerisation of lignin hence requires the breaking of several types of bonds/linkages, which — if successful—will generate a complex mixture that is highly affected by the method/approach of depolymerisation adopted. In addition, and unlike cellulose, lignin is characterised by a diversity of distinct and chemically unalike bonding motifs, each requiring different cleavage conditions when selective depolymerisation and conversion attempts are targeted [2]. To add an insight, upon employing hydrothermal treatments for this refractory structure and aside from the phenolic monomers produced, a substantial amount of oligomeric compounds are also obtained, like dimers, trimers and high-molecular-mass oligomers, suggesting notable variations in the product. Due to such difficulties in the deconstruction step itself as well as in the product separation and based on the great potential of such a durable biopolymer in future renewable energy industries, lignin depolymerisation has become the gold-rush for numerous academics and key players within the bioenergy field worldwide [10]. In light of this, finding an efficient and cost-effective way of depolymerising such complex macromolecule into low-molecular-weight fragments that could be further utilised is thus pressing.
Studies on base-catalysed depolymerisation of lignin to produce phenolic compounds, in particular, have been conducted in various research efforts and are indeed current mainstay research [11-17]. The fact that soda is already adopted within the pulping processes promotes the potential of employing such homogeneous alkaline catalysis - from a process integration perspective. It is thus considered one of the primary stateof-the-art technologies available for lignin depolymerisation together with acid-catalysed depolymerisation, pyrolysis, gasification, hydrotreating, liquid-phase reforming and biodegradation. Miller et al. [13] investigated a collection of bases for depolymerising lignin and lignin model compounds within rapidly heated micro-autoclaves in the presence of alcohols. In their study, it was concluded that stronger bases, like $\mathrm{CsOH}$, $\mathrm{KOH}$ and $\mathrm{NaOH}$, are capable of producing higher yields of low-molecular-weight products than weaker bases, e.g. $\mathrm{LiOH}$ and $\mathrm{Ca}(\mathrm{OH})_{2}$. Shabtai and co-workers [14] patented a two-step process for converting lignin into a suitable blending component for gasoline, where the initial step involved the basecatalysed lignin depolymerisation. This was subsequently followed by catalytic hydrodeoxygenation to generate gasoline-range aromatic fuels.

Going forward, Mahmood et al. [16] were successfully able to depolymerise kraft lignin into polyols through direct hydrolysis and using $\mathrm{NaOH}$ as a catalyst. The workable operating conditions seemed to be at $250^{\circ} \mathrm{C}, \mathrm{NaOH} /$ lignin ratio of 0.28 with $20 \mathrm{wt} \%$ substrate concentration and a 1-h reaction time, producing a suitable polyols replacement stream for rigid polyurethane foam synthesis. In another recent attempt, Dos Santos et al. [15] were able to produce bio-oil from basecatalysed depolymerisation of organosolv lignin at $300{ }^{\circ} \mathrm{C}$ and 40 min reaction time. The generated bio-oil solution was applied on wood and exhibited a good performance in improving its fungal resistance, introducing a new application for the produced reaction mixture from such process. In a very recent research effort by Katahira and co-workers [12], basecatalysed depolymerisation was applied on residual lignin-
Fig. 1 Structures of the three primary building blocks of lignin (monolignols). The numbering of carbon atoms in the benzene ring and notation on the aliphatic propylene side chain are also depicted

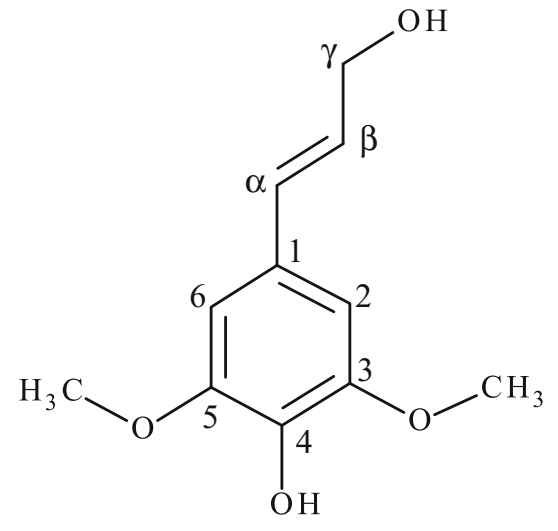

Sinapyl alcohol

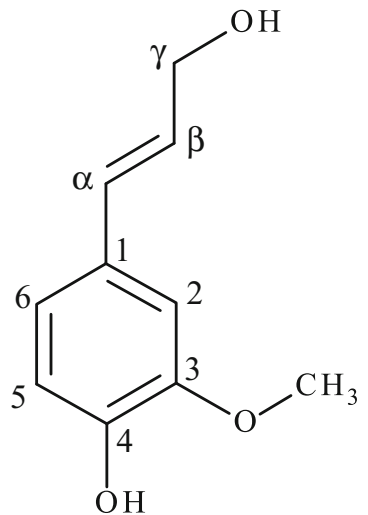

Coniferyl alcohol

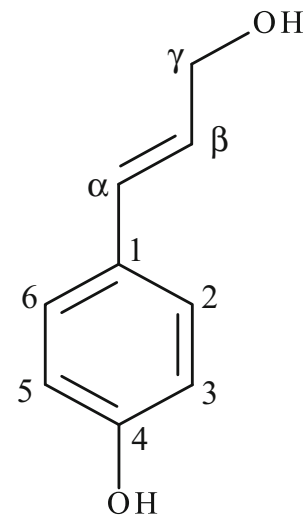

p-Coumaryl alcohol 
rich biorefinery streams with $10 \mathrm{wt} \%$ solid loading, up to $4 \mathrm{wt} \% \mathrm{NaOH}$ concentration, 40 min retention time and at temperature ranges of $270-330{ }^{\circ} \mathrm{C}$. The study introduced some light on the viability of producing considerable yields of low-molecular-weight aromatics from such substrates and its potential valorisation and further upgrading to chemicals and fuels. It is worth to point out that the aforementioned research efforts were conducted in batch operation mode.

Certainly, repolymerisation reactions are believed to be one of the principal problems in the production of phenolic compounds during lignin depolymerisation. It is thus crucial to take into account decreasing the rate of repolymerisation/ oligomerisation reactions during base-catalysed lignin depolymerisation, raising a key issue towards enhancing the yields of the low-molecular-weight products. Targeted research efforts $[11,18,19]$ have tackled such problem via introducing capping agents in water with the aim of entrapping the available reactive fragments (e.g. phenolic compounds, formaldehyde) and masking the active sites $\left(\mathrm{C}_{\alpha}\right.$ present in the original lignin structure). In a very interesting research contribution, Roberts et al. [18] applied the usage of boric acid as a capping agent in such reaction with the aim of enhancing the product yields and suppressing the addition and condensation reactions of the initially formed products. The boric acid performed well as a protective agent during the hydrothermal lignin depolymerisation, minimising concurrent oligomerisation and polymerisation reactions and leading to higher product yields beyond $85 \%$. Toledano et al. [11] also managed to overcome this problem via employing boric acid and phenol as capping agents and testing their nature and influence on base-catalysed lignin depolymerisation. An interesting point and provided that the capping agent/lignin ratio was optimised, phenol, in specific, appeared to boost the overall process through not only avoiding the repolymerisation phenomena but also favouring the production of more monomeric phenolic compounds, such as catechols, cresols and ferulic acid.

The use of H-donating reagents/solvents, such as formic acid and other stabilising compounds/alcohols have also been investigated in order to suppress the char formation problem and enhance the operation [20-23]. Gosselink et al. [20] tackled the addition of formic acid as hydrogen donor and experienced an increase in the yield of monomeric aromatics by stabilising the aromatic radicals, up to $12 \%$ based on lignin. Huang et al. [22] adopted the formic acid as well as an in situ hydrogen source during the depolymerisation of kraft lignin. It was demonstrated in this study that the formic acid is a more reactive hydrogen source than external hydrogen towards the reductive depolymerisation of technical lignin streams. It is speculated that the formic acid, in particular, is generated during the lignin pulping process (e.g. organosolv) and therefore can viably serve as an internal hydrogen donor with better hydrogen transfer protocols [24]. It is noteworthy that although lots of efforts have been made to develop efficient methods for lignin depolymerisation, the step to take this fundamental research to further industrial applications remains a major challenge.

The objective of this study is to screen the best reaction conditions for depolymerisation of technical lignin streams using base catalysis in continuous-mode operation, yielding the highest quantity of low-molecular-mass phenolic compounds. To the best of the authors' knowledge, the present contribution is the first of its nature to address the basecatalysed lignin depolymerisation using a novel continuousmode operation system, identifying new regimes/conditions for such reaction. It is worth noting that most lignin depolymerisation reactions/processes are performed at temperatures of $250-650^{\circ} \mathrm{C}$, with or without catalysts, as critically reviewed by Zakzeski et al. [25] and Li et al. [4]; here in this paper, new milder conditions are introduced for such reaction typically below $240{ }^{\circ} \mathrm{C}$ in a continuous-mode operation and without exploiting any capping agents or hydrogen donors. The experimental/reaction conditions tested implied a temperature range of $170-250{ }^{\circ} \mathrm{C}$, a pressure of 130 bar and residence time span of 1-4 min. Storage stability tests were investigated and product separation tasks were conducted on the obtained reaction mixture prior analysis. The lignin substrate and generated/depolymerised products were finally characterised using elementary analysis, size exclusion chromatography (SEC), nuclear magnetic resonance spectroscopy (NMR) and supercritical fluid chromatography-diode array detector-tandem mass spectrometry (SFC-DAD/MS).

\section{Materials and methods}

\subsection{Materials}

The commercial kraft lignin, Indulin AT, which is a pine softwood lignin that is precipitated from black liquor of linerboard-grade pulp, was obtained as dry powder from MeadWestvaco Corporation (Charleston Heights, SC) [26]. In this work, Indulin AT was used as the starting raw material in all experiments. This lignin stream has a moisture content of about $4.5 \mathrm{wt} \%$ and ash content of $3.5 \mathrm{wt} \%$. The sample was analysed for its elemental composition at BELAB AB in Norrköping, Sweden; and full proximate, ultimate and compositional analyses for the lignin sample are reported in Table 1. The higher heating value (HHV) of the lignin sample was calculated based on the composition of main elements through an applied formula [27] with more than $90 \%$ predictions accuracy and in the range of $\pm 5 \%$ error (i.e. HHV (MJ/ $\mathrm{kg})=-1.3675+0.3137 \mathrm{C}+0.7009 \mathrm{H}+0.0318 \mathrm{O} *$ ). The elemental compositions are in weight percentage, and $\mathrm{O}^{*}$ is the sum of the contents of oxygen and other elements in the organic matter, including sulphur and nitrogen. 
Table 1 Elementary analysis of Indulin AT lignin sample. The values are reported on a dry lignin basis, with available uncertainty ( $95 \%$ confidence interval). The oxygen content is calculated by difference

\begin{tabular}{ll}
\hline Analysis & Indulin AT lignin \\
\hline Proximate analysis & \\
Ash (\%) & 3.5 \\
Volatiles (\%) & 62.8 \\
Fixed carbon (\%) & 33.7 \\
Moisture (\%) & 4.5 \\
HHV (MJ/kg & 23.6 \\
Ultimate analysis (\% dry basis) \\
C (\%) & $63.9^{\mathrm{a}}$ \\
H (\%) & $5.8^{\mathrm{a}}$ \\
$\mathrm{N}(\%)$ & $0.7^{\mathrm{a}}$ \\
$\mathrm{S}(\%)$ & $1.5^{\mathrm{b}}$ \\
$\mathrm{O}(\%)$ & 24.6 \\
\hline
\end{tabular}

${ }^{a}$ Measured according to the SS-EN 15104 norm using a LECO Truspec CHN instrument

${ }^{\mathrm{b}}$ Determined according to the SS-EN 15289 method with a LECO SC-144DR sulphur and carbon analyser

The feed was prepared prior to each experimental run and was composed typically of $5 \mathrm{wt} \%$ lignin substrate, $5 \mathrm{wt} \%$ $\mathrm{NaOH}$ and $90 \mathrm{wt} \%$ deionised (DI) water. Vacuum filtration for the feed was performed to ensure that no precipitation could occur in the pump and to prevent clogging problems within the pressure valve further after reaction. Sodium hydroxide, hydrochloric acid (37\%) and all other chemicals were purchased from Sigma-Aldrich Sweden AB.

\subsection{Depolymerisation apparatus and procedure}

The base-catalysed lignin depolymerisation experiments were performed in a new-tailored continuous flow reactor (CFR), and a schematic diagram of the designed setup is presented in Fig. 2.
The system is composed of a Gilson 307 HPLC pump (Middleton, WI, USA), a Salamander tubular CFR reactor (Cambridge Reactor Design Ltd., Cottenham, UK) with a heated volume of 18 and $50 \mathrm{~mL}$ total system volume (ensured in safe reaction zone, i.e. $\max P \times V=8.15$ bar $\mathrm{L}<$ 20 ), and a specially assembled pressure control valve from nickel-molybdenum-chromium superalloy (Hastelloy C276; UNS N10276). The same material used in manufacturing the valve-Hastelloy C276-was also used for the reactor tubes to replace normal stainless steel tubes, offering excellent corrosion resistance in such severely basic environment. A filter was installed after the cooler in order to protect the pressure control valve from any char depositions if any. Before starting each experimental run, the setup was heated up and pressurised to the targeted operating conditions, keeping a continuous flow of DI water within the whole system. The feed was then continuously pumped to the reactor at volumetric flowrates of 10 , 5 and $2.5 \mathrm{~mL} / \mathrm{min}$, respectively. These mixtures flowing through the reactor system typically correspond to residence times in the heating zone of about 1,2 and $4 \mathrm{~min}$, respectively. The experimental parameters and conditions are summarised in Table 2.

The experiments were conducted at different screening temperatures ranging from 170 to $250{ }^{\circ} \mathrm{C}$, with $10{ }^{\circ} \mathrm{C}$ intervals, at pressures around 130 bar and retention times span of 1-4 min. For the reaction parameters, the temperature was set using a Eurotherm temperature controller (Eurotherm, Ashburn, Virginia, USA) and the pressure was adjusted through a backpressure regulator (Bronkhorst High-Tech B.V., Ruurlo, Netherlands). After exiting the reaction zone and expanding the product mixture to atmospheric pressure, the liquid products were continuously collected separately during the different operating conditions for workup and analysis purposes. The collected product samples were also divided into two cuts for storage stability testing, where one moiety was stored at room temperature and the other at $4{ }^{\circ} \mathrm{C}$.
Fig. 2 Schematic diagram of the experimental setup

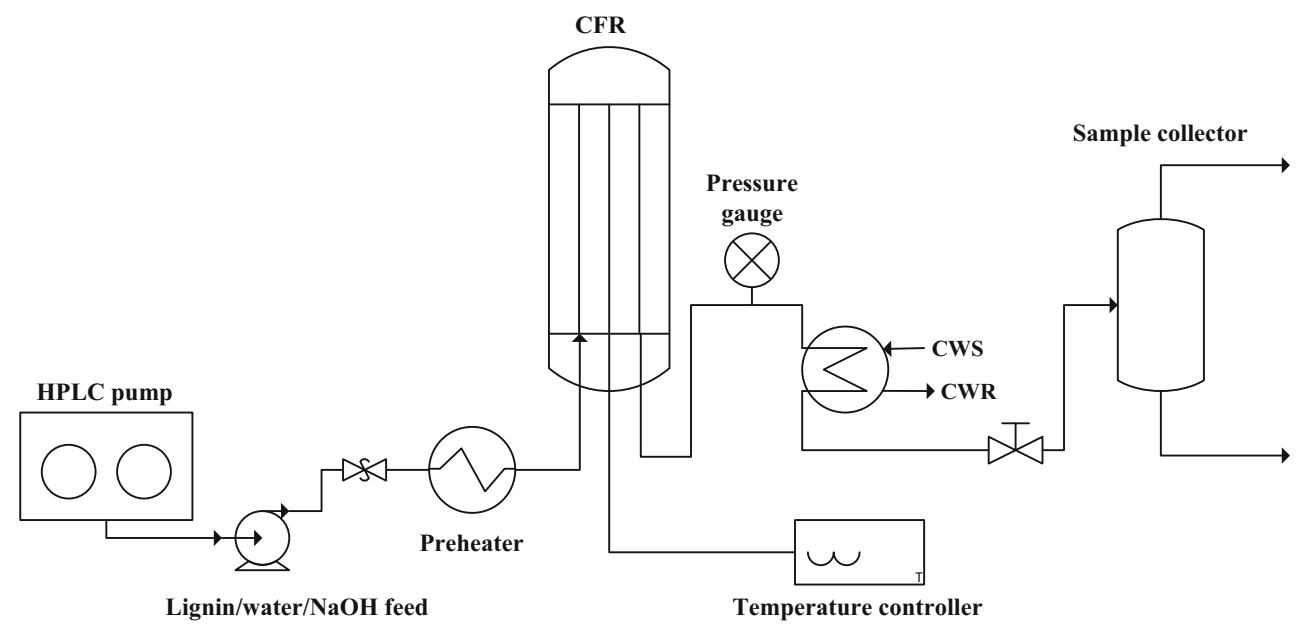


Table 2 Lignin

depolymerisation experimental conditions

\begin{tabular}{ll}
\hline Parameter & Range tested \\
\hline $\mathrm{NaOH}$ (wt $\%)$ & 5 \\
Lignin loading (wt\%) & 5 \\
$\mathrm{pH}$ range & $12-14$ \\
Temperature $\left({ }^{\circ} \mathrm{C}\right)$ & $170-250$ \\
Pressure (bar) & $120-130$ \\
Time (min) & $1-4$ \\
\hline
\end{tabular}

\subsection{Molecular weight distribution}

Following an established method [28], the molecular weight distributions of Indulin AT lignin as well as depolymerised lignin samples were determined and screened using a SEC system. The system used for the size screening studies was a Waters 600E chromatography system (Waters, Milford, MA, USA) equipped with a Waters 2414 refractive index (RI) detector, a Waters $486 \mathrm{UV}$ absorbance detector and an analytical column packed with $30 \mathrm{~cm}$ of Superdex 30 and $30 \mathrm{~cm}$ of Superdex 200 (GE Healthcare, Uppsala, Sweden). The column was operated at ambient temperature and eluted with $125 \mathrm{mM} \mathrm{NaOH}$ solution (analytical grade) as the mobile phase at a flowrate of $1.0 \mathrm{~mL} / \mathrm{min}$. Calibration was performed using polyethylene glycol (PEG) standards ranging from 400 to $35,000 \mathrm{~g} / \mathrm{mol}$ in the eluent (Merck Schuchardt OHG, Hohenbrunn, Germany). The samples were dissolved/diluted at concentrations of about $1 \mathrm{mg} / \mathrm{mL}$ in the eluent, and the solutions were further filtered using a $0.2-\mu \mathrm{m}$ filter (Schleicher and Schuell, Dassel, Germany) in order to remove any suspended matter; and $500 \mu \mathrm{L}$ from the filtered solution was lastly injected into the SEC system. Due to the comparison with PEG standards, both the molecular weight and the molecular number should be considered relative. For the storage stability tests, the feed mixture and the depolymerised lignin product samples $\left(240\right.$ and $\left.250{ }^{\circ} \mathrm{C}\right)$ were stored at different temperatures for periods of 1, 2 and 3 months before finally being analysed using the SEC system.

\subsection{Characterisation approach}

For detailed analysis of substrate and produced products, a workup procedure was developed for better characterisation results where separation tasks were involved. Basically, the product reaction mixture was first acidified with $6 \mathrm{M} \mathrm{HCl}$ to $\mathrm{pH} 1$, which resulted in the precipitation of insoluble fragments. The supernatant - generated after acidification-was saved after centrifugal separation and extracted three times with ethyl acetate as extraction solvent. The ethyl acetate extract was then transferred quantitatively to a glass tube and evaporated under a gentle $\mathrm{N}_{2}$ flow, where residue $A$ (low-molecular-weight degradation products) was obtained. Residue $A$ was finally stored for further SFC-MS analysis and subjection to a set of $2 \mathrm{D}{ }^{1} \mathrm{H}-{ }^{13} \mathrm{C}$ HSQC NMR experiments. The acidinsoluble lignin product (residue $B$ ) originated after acidification was washed more than three times with DI water, freezedried for $24 \mathrm{~h}$ and stored for other analysis set.

For the SFC-DAD/MS analysis, residue $A$ was first dissolved in $1 \mathrm{~mL}$ of methanol. The solution was then stored in a freezer at $-80{ }^{\circ} \mathrm{C}$ before being analysed. An ultra-highperformance supercritical fluid chromatography coupled with quadrupole-time-of-flight mass spectrometry method for the separation/analysis of major lignin-derived phenolic compounds was adopted in this sense, following a newly developed procedure [29]. Chromatographic separation and detection of the sample was performed with a Waters UltraPerformance Convergence Chromatography System (Milford, MA, USA) coupled to a Waters Xevo G2 Q-TOF mass spectrometer with electrospray ionisation (ESI) and atmospheric pressure chemical ionisation (APCI) (Waters, MS Technologies, Manchester, UK). The column employed was Waters Torus DIOL $(1.7 \mu \mathrm{m}, 3 \times 100 \mathrm{~mm})$ for target compounds separation. The mobile phase was composed of mainly $\mathrm{CO}_{2}$ gas and methanol as co-solvent. The column temperature was set at $50{ }^{\circ} \mathrm{C}$, and the pressure of the backpressure regulator was adjusted at 130 bar. A binary gradient elution program was employed with supercritical $\mathrm{CO}_{2}$ and methanol as solvents. The mobile phase gradient started with 100:0 $\left(\mathrm{CO}_{2} / \mathrm{CH}_{3} \mathrm{OH}\right.$ vol\% $)$ and ramped up to $91.5-8.5 \%$ of $\mathrm{CO}_{2}$ solvent in $2.5 \mathrm{~min}$, then ramped up to $75-25 \%$ of $\mathrm{CO}_{2}$ solvent in $3 \mathrm{~min}$ and hold for $1 \mathrm{~min}$ and lastly decreased to the starting composition after $0.5 \mathrm{~min}$. Ammonia $(5 \mathrm{mM})$ in methanol was used as a makeup solvent, $0.2 \mathrm{~mL} / \mathrm{min}$. The data acquisition was done in a negative ion ESI mode. The data acquisition range was $m / z, 150-800$. The capillary voltage was $3 \mathrm{kV}$, the extraction cone voltage was $4 \mathrm{~V}$ and the sampling cone voltage was $20 \mathrm{~V}$. The source temperature was $120^{\circ} \mathrm{C}$, and the desolvation temperature was $600{ }^{\circ} \mathrm{C}$ with the desolvation gas flowrate of $1200 \mathrm{~L} / \mathrm{h}$. The cone gas flowrate was $40 \mathrm{~L} / \mathrm{h}$.

A set of ${ }^{1} \mathrm{H}$ and $2 \mathrm{D}{ }^{1} \mathrm{H}-{ }^{13} \mathrm{C}$ HSQC NMR experiments for lignin and depolymerised products at $25^{\circ} \mathrm{C}$ were acquired on Agilent VNMRS NMR spectrometers (Santa Clara, CA, USA) operating at 500 and $600 \mathrm{MHz}$, both equipped with a 5-mm HCN probe with triple-axis gradients. Typically, $50 \mathrm{mg}$ of sample was dissolved in $0.5 \mathrm{~mL}$ of deuterated dimethyl sulfoxide- $d_{\sigma}$ (DMSO- $d_{6}$ ) after stirring. Chemical shifts were referenced to the residual solvent signal at 2.5 and $39.5 \mathrm{ppm}$ for ${ }^{1} \mathrm{H}$ and ${ }^{13} \mathrm{C}$, respectively. The ${ }^{1} \mathrm{H}$ spectra were acquired using a $90^{\circ}$ pulse angle, an acquisition time of $2 \mathrm{~s}$, a relaxation delay of 2 s, 32 scans for the lignin sample and 4 scans for depolymerised products. The HSQC experiments were acquired using the Agilent pulse sequence gHSQC, a relaxation delay of $2 \mathrm{~s}$, an acquisition time of $150 \mathrm{~ms}$ and 256 complex data points in the indirect dimension but were otherwise not acquired identically for the two samples. For the sample of depolymerised lignin residue, an edited experiment was used, 
with spectral widths of 13.4 and $170 \mathrm{ppm}$ for the ${ }^{1} \mathrm{H}$ and ${ }^{13} \mathrm{C}$ dimensions, respectively, and 64 transients. The total experimental time was $20 \mathrm{~h}$. For the lignin sample, the experiment was run without editing, in order to maximise the signal intensities, with spectral widths of 12 and $170 \mathrm{ppm}$ for the ${ }^{1} \mathrm{H}$ and ${ }^{13} \mathrm{C}$ dimensions, respectively, and 80 transients. The total experimental time was $25 \mathrm{~h}$. The spectra were processed using VnmrJ software (v4.2).

\section{Results and discussion}

\subsection{Size exclusion chromatography}

Size exclusion chromatography (SEC) was performed to assess changes in the distribution of molecular mass of lignin samples before and after depolymerisation at temperatures ranging from 170 to $250{ }^{\circ} \mathrm{C}$ and, initially, with the flowrate of $10 \mathrm{~mL} / \mathrm{min}$. Figure $3 \mathrm{a}, \mathrm{b}$ shows the corresponding $\mathrm{UV}$ absorbance chromatograms and refractive index elution profiles of different lignin samples, respectively. A flowrate of $10 \mathrm{~mL} / \mathrm{min}$ was employed, corresponding to residence time of approximately $1 \mathrm{~min}$ in the reactor. As depicted in Fig. 3a, the initial molecular weight distribution profile of Indulin AT lignin generally distributed in a distinct wide region from 1 to $100 \mathrm{kDa}$ with a peak around $4 \mathrm{kDa}$, illustrating that the main parts of Indulin AT are heavy compounds (heavier than $1 \mathrm{kDa}$ ). In addition, a small peak curve was also shown at approximately $0.9 \mathrm{kDa}$, representing a fraction of lowmolecular-weight oligomers. The molecular weight distribution of the products gradually shifts towards lower molecular weight components with the increasing depolymerisation temperature (i.e. from 170 to $250{ }^{\circ} \mathrm{C}$ ). The heaviest components with a molecular weight higher than $10 \mathrm{kDa}$ almost disappeared when the temperature increased to $250^{\circ} \mathrm{C}$. It was noted that the bimodal curve distribution around $200-400 \mathrm{Da}$, which represents basically monomers and dimers, appeared when the temperature was above $220^{\circ} \mathrm{C}$. The higher depolymerisation
Fig. 3 Molecular weight distributions of Indulin AT and depolymerised lignin products, measured as a UV absorbance at $280 \mathrm{~nm}$ and $\mathbf{b}$ refractive index. The depolymerisation reaction sets were performed at temperatures ranging from 170 to $250{ }^{\circ} \mathrm{C}$ with the flowrate of $10 \mathrm{~mL} / \mathrm{min}$ (reaction time of $1 \mathrm{~min}$ )
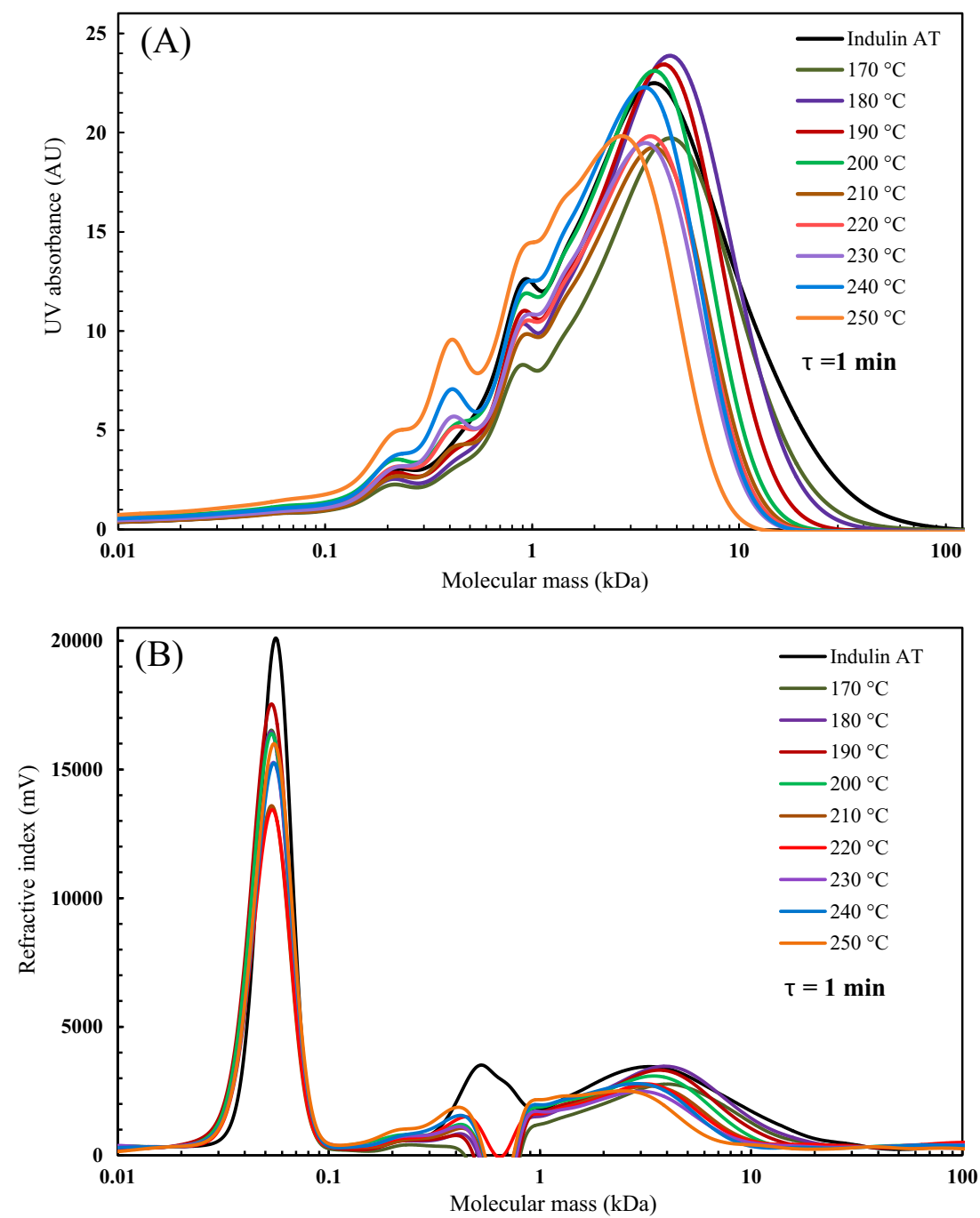
temperature yielded in turn substantially more monomers and dimers. Here, the lowest molecular mass distribution and the clearest peak at 200-400 Da were observed in the highest temperature $\left(250{ }^{\circ} \mathrm{C}\right)$. The results at this juncture suggest that a more complete depolymerisation of lignin takes place with higher temperatures for such operation.

On the other hand, as shown in Fig. 3b, all the lignin samples had refractive index (RI) peaks at 40-70 Da, which comes from the sodium hydroxide. This illustrates that the hydrothermal depolymerisation reaction at such conditions is a sodium hydroxide consuming process. Such phenomenon was found to be comparable with other similar studies [25, 30], indicating/ confirming that a high consumption of base is to be expected upon depolymerising lignin under alkaline conditions. Also during operation, the $\mathrm{pH}$ was lowered by two orders of magnitude confirming the consumption of alkali. A wide peak at about $1-10 \mathrm{kDa}$ was also observed in the RI profiles (Fig. $3 b)$. The peak weakened slightly with the increasing depolymerisation temperature, indicating the decrease of the molecular weight of Indulin AT lignin after depolymerisation.
Towards a better understanding of the reaction mechanism/ behaviour and the CFR system performance, another screening attempt was performed at the same temperature range (170-250 ${ }^{\circ} \mathrm{C}$ ) but with a lower feed flowrate of $5 \mathrm{~mL} / \mathrm{min}$, corresponding to a residence time of approximately $2 \mathrm{~min}$. The UV absorbance chromatograms and the refractive index elution profiles for the lignin and depolymerised lignin samples at the aforementioned operating conditions are depicted as Fig. 4a, b, respectively.

Compared with the flowrate of $10 \mathrm{~mL} / \mathrm{min}$, a more thorough depletion of components over $10 \mathrm{kDa}$ was observed from the temperature of $170{ }^{\circ} \mathrm{C}$ (Fig. 4a). Another stage of reduction happened when the temperature increased up to $190{ }^{\circ} \mathrm{C}$. Accordingly, the peaks at around $400 \mathrm{Da}$ and $1 \mathrm{kDa}$ just get more and more distinct and higher above $190{ }^{\circ} \mathrm{C}$. This represents a substantial increase in the production of monomers, dimers and other low-molecular-weight oligomers with the depolymerisation of Indulin AT kraft lignin. However, compared with $240{ }^{\circ} \mathrm{C}$, an increase of the peak value at about $3 \mathrm{kDa}$ was observed in the temperature of $250{ }^{\circ} \mathrm{C}$. For the
Fig. 4 Molecular weight distributions of Indulin AT and depolymerised lignin products, measured as a UV absorbance at $280 \mathrm{~nm}$ and $\mathbf{b}$ refractive index. The depolymerisation reaction sets were performed at temperatures ranging from 170 to $250{ }^{\circ} \mathrm{C}$ with the flowrate of $5 \mathrm{~mL} /$ $\min$ (reaction time of $2 \mathrm{~min}$ )
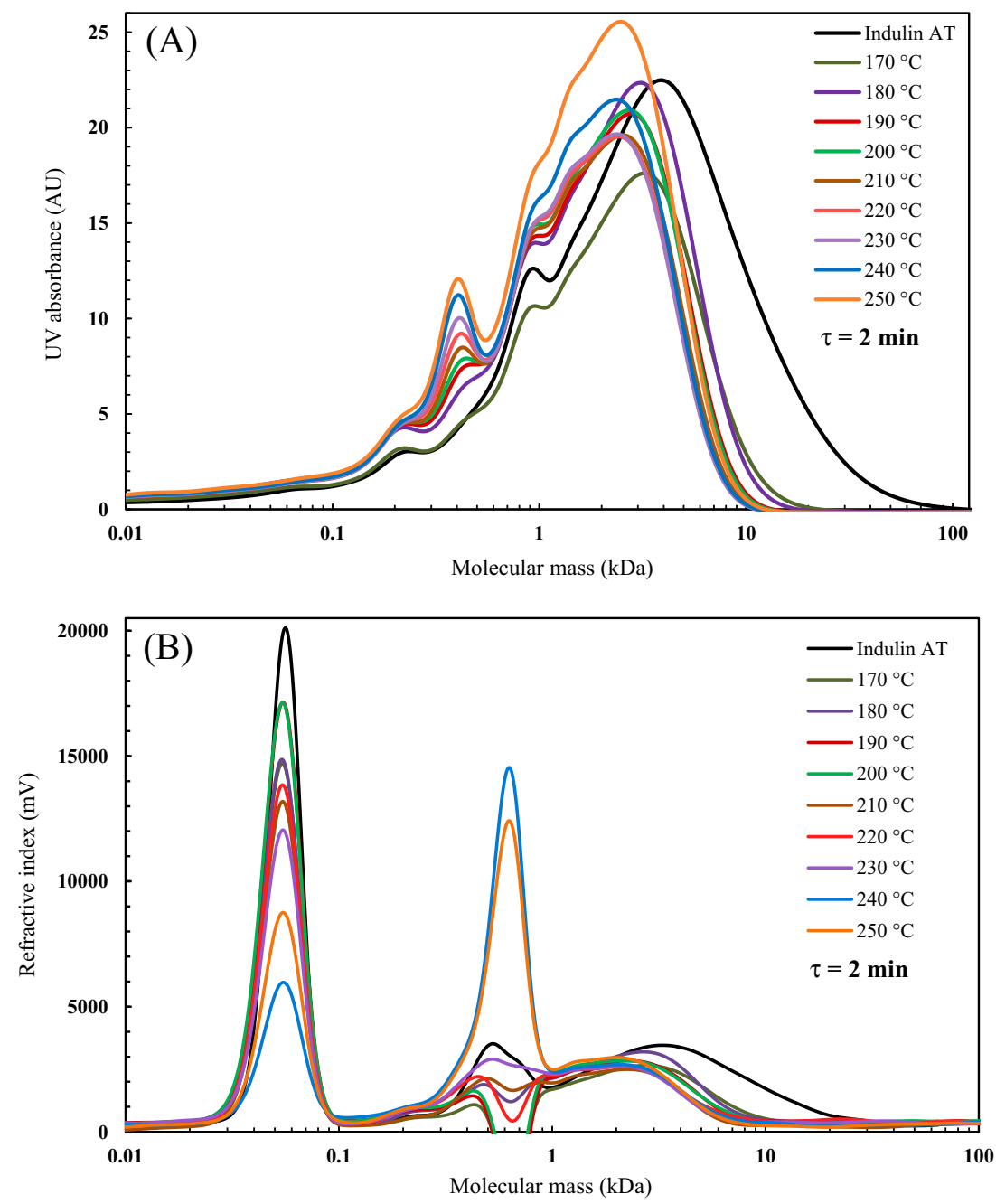
Fig. 5 Molecular weight distributions of Indulin AT and depolymerised lignin products, measured as a UV absorbance at $280 \mathrm{~nm}$ and $\mathbf{b}$ refractive index. The depolymerisation reaction sets were performed at temperatures ranging from 170 to $240{ }^{\circ} \mathrm{C}$ with the flowrate of $2.5 \mathrm{~mL} / \mathrm{min}$ (reaction time of $4 \mathrm{~min}$ )

Fig. 6 Comparison of the molecular weight distributions of Indulin AT and depolymerised lignin products at temperatures of 230 and $240{ }^{\circ} \mathrm{C}$ (measured as UV absorbance at $280 \mathrm{~nm}$ ) with the flowrate of 10,5 and $2.5 \mathrm{~mL} / \mathrm{min}$
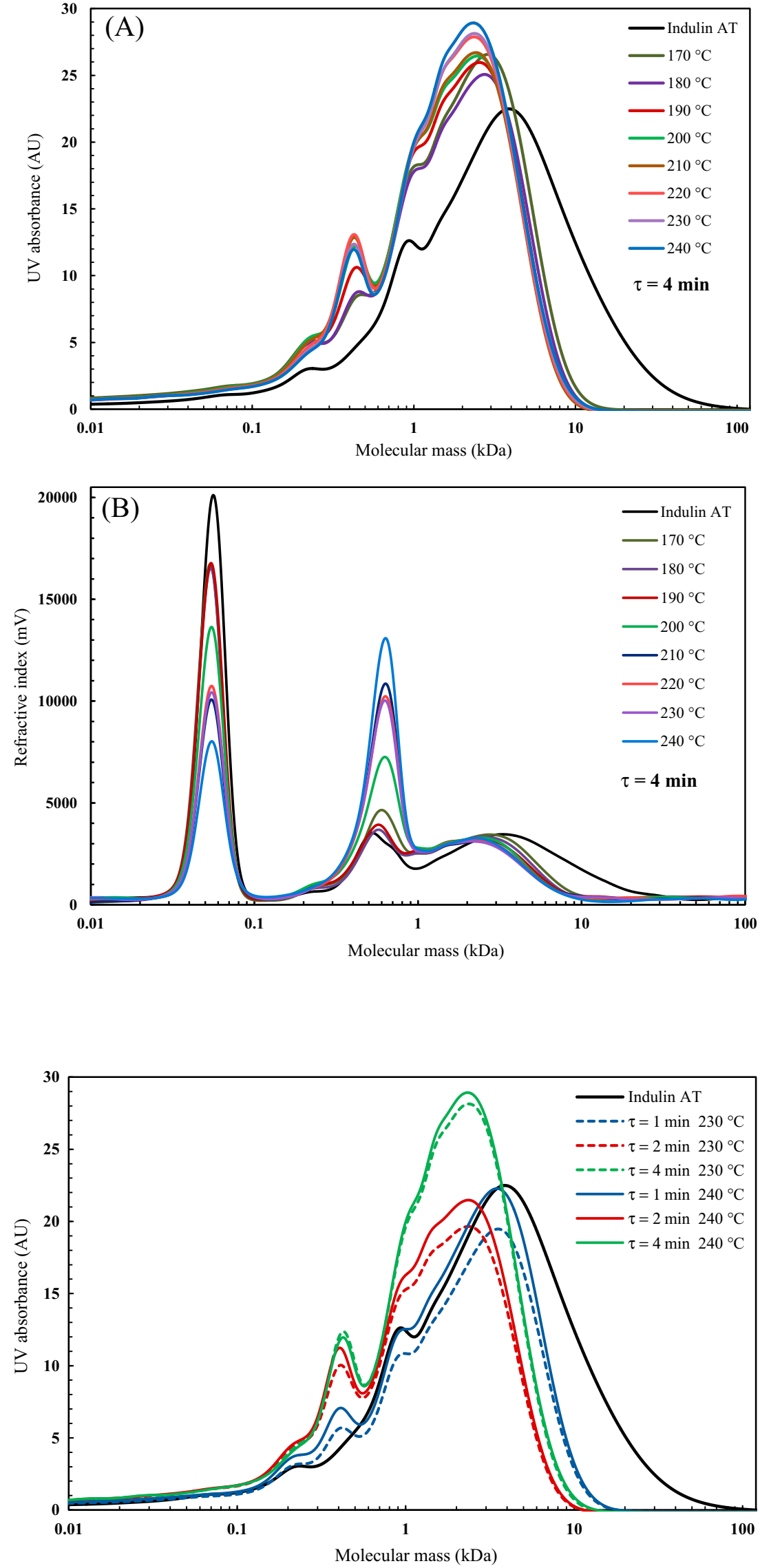
Fig. 7 Molecular weight distribution variations of Indulin AT lignin at different storage times testing the substrate stability

Fig. 8 Molecular weight distributions of depolymerised lignin samples at different storage times (measured as UV

absorbance at $280 \mathrm{~nm}$ ). The depolymerisation was performed with $10 \mathrm{~mL} / \mathrm{min}$ flowrate at the temperatures 240 and $250^{\circ} \mathrm{C}$. The samples were stored at a room temperature (RT) and b $4{ }^{\circ} \mathrm{C}$ for periods of 2 and 3 months, respectively
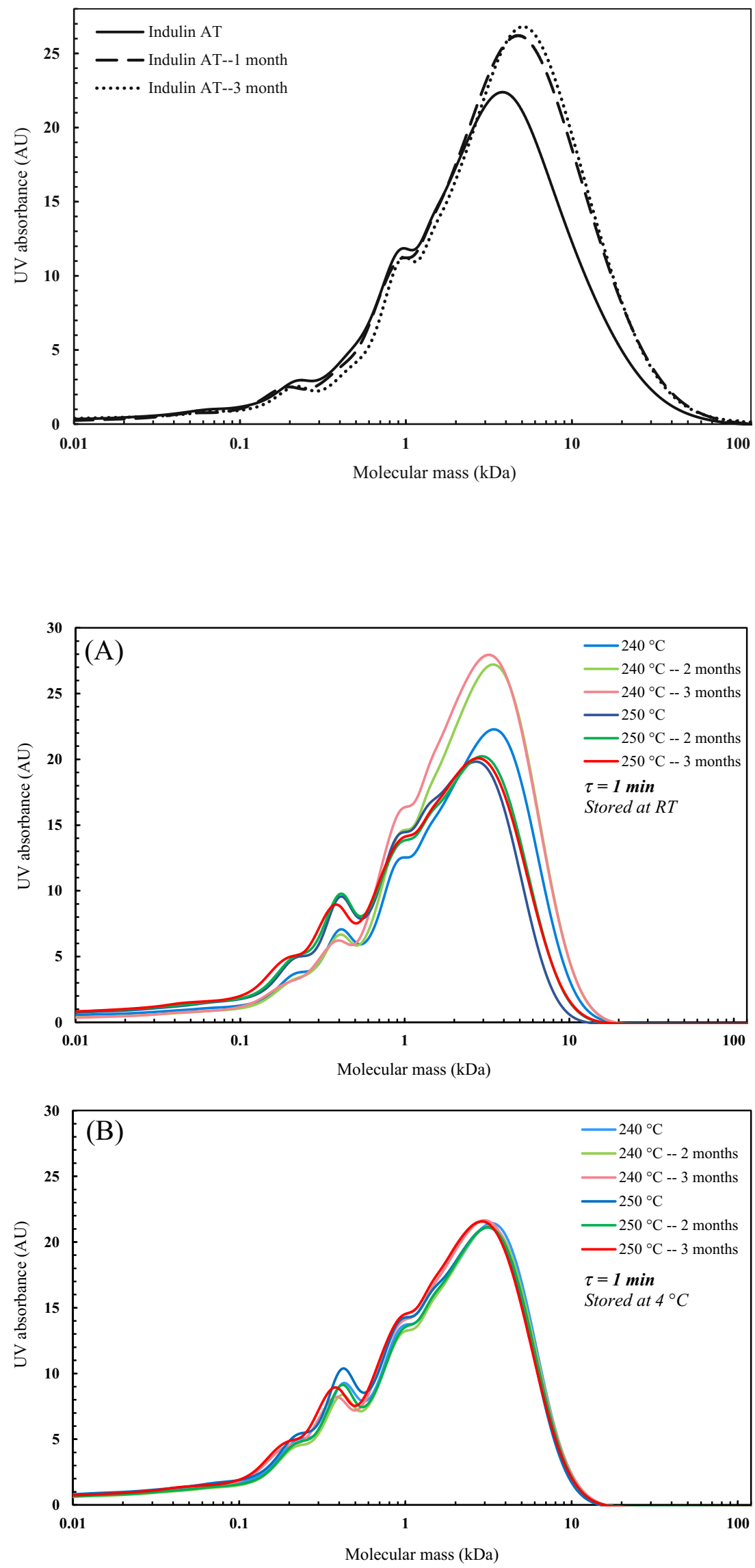
experiments performed at $5 \mathrm{~mL} / \mathrm{min}$ flowrate, char was experienced/formed at the temperature of $250{ }^{\circ} \mathrm{C}$. The flowrate suddenly dropped, and the reactor tubing was plugged upon raising the temperature and feed flowrate, hindering any flow of feed solution throughout the CFR system. Therefore, temperature of $250{ }^{\circ} \mathrm{C}$ is not recommended for the continuous depolymerisation of Indulin AT lignin at a residence time of $2 \mathrm{~min}$.

Figure $4 \mathrm{~b}$ clearly shows the consumption of $\mathrm{NaOH}(40$ $70 \mathrm{kDa}$ ), i.e. lower refractive index exhibited, with the increased depolymerisation temperature. The results from the experiments at 240 and $250{ }^{\circ} \mathrm{C}$ displayed RI peaks at approximately 0.5 $0.8 \mathrm{kDa}$ which indicates the production of low-molecularweight components tentatively at such conditions (Fig. 4b). In addition, the RI peaks at $1-10 \mathrm{kDa}$ obviously decreased with the increasing depolymerisation temperature $\left(170-250{ }^{\circ} \mathrm{C}\right)$, which indicates that more cleavage of the chemical bonds in the lignin molecules occurred under this operating conditions.
Final set of depolymerisation experiments was performed at a flowrate of $2.5 \mathrm{~mL} / \mathrm{min}$ in the temperature range from 170 to $240{ }^{\circ} \mathrm{C}$. At $2.5 \mathrm{~mL} / \mathrm{min}$ feed flowrate, the residence time in the reactor was estimated to be $4 \mathrm{~min}$. Under this condition, the heavy components with molecular weight higher than $10 \mathrm{kDa}$ were totally depolymerised at different temperatures from 170 to $240{ }^{\circ} \mathrm{C}$ (Fig. 5a). There is also a remarkable growth in the amount of components with molecular mass between 1 and $5 \mathrm{kDa}$. A trend confirmed that UV absorbance and RI response increase with increased temperature was observed. However, no clear trend was experienced upon increasing the residence time, indicating that repolymerisation could be an issue at $\tau$ of 4 min (Fig. 5) in comparison to $\tau$ of 2 min (Fig. 4). More distinct peaks at $0.2-1 \mathrm{kDa}$ were exhibited in UV absorbance and refractive index, which illustrates the production of more monomers, dimers and/or other oligomers (Fig. $5 \mathrm{a}, \mathrm{b}$ ).

To gain more comparative insights between different attained results, Fig. 6 was plotted showing different SEC
Fig. 9 Molecular weight distributions of depolymerised lignin samples at different storage times (measured as UV absorbance at $280 \mathrm{~nm}$ ). The depolymerisation was performed with $5 \mathrm{~mL} / \mathrm{min}$ flowrate at the temperatures 240 and $250^{\circ} \mathrm{C}$. The samples were stored at a room temperature (RT) and $\mathbf{b} 4{ }^{\circ} \mathrm{C}$ for periods of 2 and 3 months, respectively
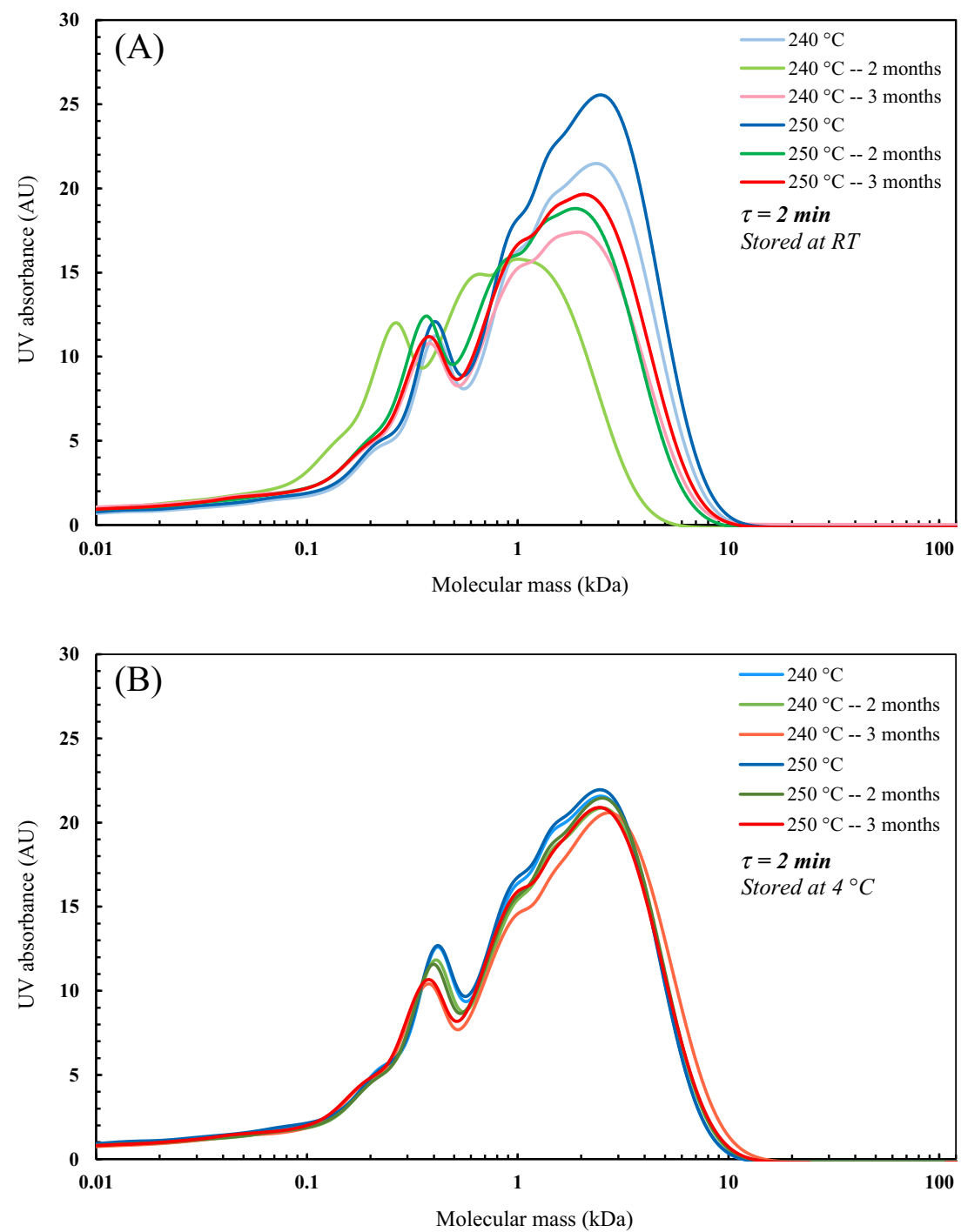
profiles recorded for the three residence times tested (1, 2 and $4 \mathrm{~min}$ ) at 230 and $240{ }^{\circ} \mathrm{C}$ (sweet depolymerisation spots). Comparing the changes encountered at different residence times under the same depolymerisation temperature, it can be deduced that longer residence time yields more low-molecularmass oligomers, which can be demonstrated from Fig. 6. However, with $4 \mathrm{~min}$ of reaction time, the repolymerisation of Indulin AT became prominent. These phenomena can be visually seen in the comparative plot, where the curves for the experiments performed at temperatures of 230 and $240{ }^{\circ} \mathrm{C}$ exhibited a slight shift to the right when the residence increased from 2 to $4 \mathrm{~min}$. The results here corresponded well with the changing of molecular mass distribution of lignin during autohydrolysis process [19]. Based on these results, for further investigation, we suggest 2 min residence time and $240{ }^{\circ} \mathrm{C}$ reaction temperature as the optimum operating conditions for Indulin AT base-catalysed depolymerisation in a continuous-mode processing.

The storage stability of lignin oils and lignin-derived biooils is a critical factor that would significantly influence the bio-oil applications - from an industrial point of view. In this context, the term "storage stability" may refer to the ability of the bio-oil to retain its initial chemical and physical properties in a relatively high temperature environment, i.e. thermal
Fig. 10 Representative base peak intensity chromatograms obtained from the SFC-MS analysis for Indulin kraft lignin (IK), depolymerised lignin residues extracted from the runs performed at $T=240{ }^{\circ} \mathrm{C}$ and $\tau=2 \min$ (A), and $T=230^{\circ} \mathrm{C}$ and $\tau=1 \min (\mathrm{X})$
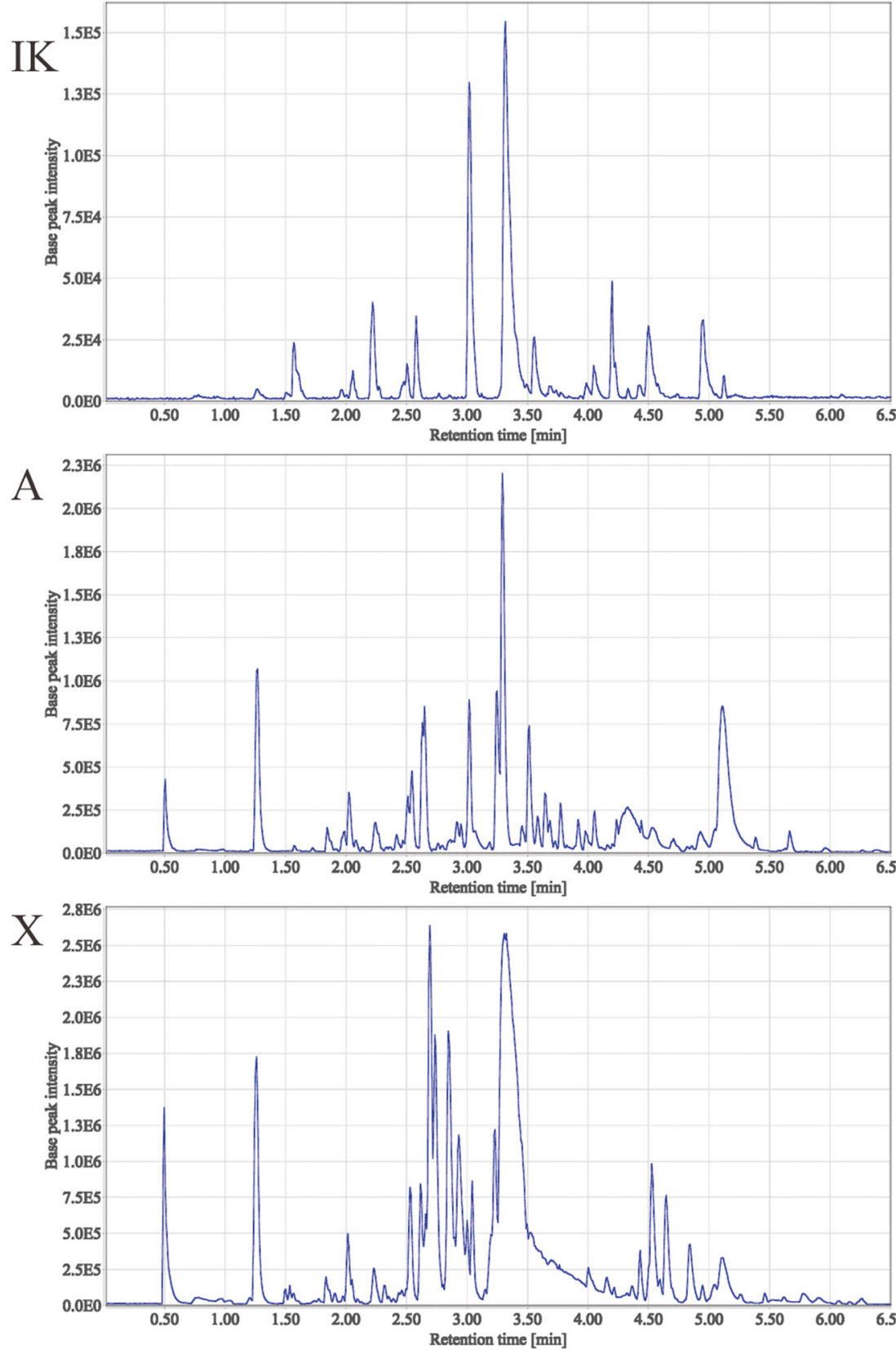
stability, or under atmospheric oxidation (oxidative stability) storage conditions [31]. The stability of lignin and ligninderived bio-oils can be tested through storage time and/or storage temperature, and here, in the present study, we investigate both the long-term storage of lignin bio-oils and the effect of storage temperature on lignin and lignin oils' properties. Figure 7 presents the molecular weight distribution variations of Indulin AT lignin starting substrate (the mixture of $5 \%$ lignin with $5 \% \mathrm{NaOH}$ and $90 \%$ distilled water) at room temperature and different storage times in order to assess the initial substrate stability. As can be seen, the lignin basic feed mixture appears to be very stable with time at normal storage conditions. This can be attributed to that there were no significant variations in size (molecular mass) encountered during this long-term storage.

The 240 and $250{ }^{\circ} \mathrm{C}$ ( $1 \mathrm{~min}$ of residence time) depolymerised lignin samples were first selected for the stability research and further compared with the ones obtained at 2 min reaction time, referring that the best depolymerisation results were experienced at these particular temperatures for such residence times. The molecular weight distribution of the first selected two depolymerised lignin samples which were stored at room temperature and refrigerated at $4{ }^{\circ} \mathrm{C}$, respectively, were compared during $0-3$ months of storage times (Fig. 8). In the condition of $4{ }^{\circ} \mathrm{C}$, the molecular weight distribution of lignin samples was rarely changed after even 3 months' storage (Fig. 8b). This means that samples stored at $4{ }^{\circ} \mathrm{C}$ of storage condition were rather stable. However, it was clear that the peaks of the depolymerised lignin samples which were stored at room temperature moved to some extent at different storage periods (Fig. 8a). One explanation can be that the depolymerised components might have been repolymerised during such storage periods. Bearing in mind the capability of produced phenolic structures to react with available reactive sites in water during lignin conversion [32], crosslinking between the active sites and generated phenolic moieties in large fragments could take place, i.e. lignin could give in turn higher-molecular-weight fragments. Similar results can be observed in the other two depolymerised lignin samples at 240 and $250{ }^{\circ} \mathrm{C}$ with the residence time of 2 min (Fig. 9). It performed/revealed almost perfect stability in the $4{ }^{\circ} \mathrm{C}$

Table 3 Most abundant chemical compounds identified by SFC-MS for Indulin kraft lignin starting material (IK), depolymerised lignin residues extracted from the runs performed at $T=240{ }^{\circ} \mathrm{C}$ and $\tau=2 \min (\mathrm{A})$ and $T=230{ }^{\circ} \mathrm{C}$ and $\tau=1 \mathrm{~min}(\mathrm{X})$

\begin{tabular}{|c|c|c|c|c|c|c|c|}
\hline \multirow[t]{2}{*}{ Sample } & \multirow[t]{2}{*}{ Compound } & \multirow{2}{*}{$\begin{array}{l}\text { Retention time } \\
(\mathrm{min})\end{array}$} & \multirow{2}{*}{$\begin{array}{l}\text { Calculated } m / z \\
\left([\mathrm{M}-\mathrm{H}]^{-}\right)\end{array}$} & \multirow{2}{*}{$\begin{array}{l}\text { Measured } m / z \\
\left([\mathrm{M}-\mathrm{H}]^{-}\right)\end{array}$} & \multirow{2}{*}{$\begin{array}{l}\text { Base peak } \\
\text { intensity }\end{array}$} & \multicolumn{2}{|c|}{$\mathrm{MS}^{2}$ fragmentation } \\
\hline & & & & & & $\mathrm{MS}^{2}$ transition & Lost fragment \\
\hline \multirow[t]{3}{*}{ IK } & $p$-Hydroxybenzaldehyde & 2.48 & 121.0290 & 121.0317 & 7.9E3 & $121 \rightarrow 92$ & $\mathrm{CHO}$ \\
\hline & Vanillic acid & 3.29 & 167.0345 & 167.0356 & $1.5 \mathrm{E} 5$ & $\begin{aligned} & 167 \rightarrow 152 \\
& 167 \rightarrow 123 \\
& 167 \rightarrow 108\end{aligned}$ & $\begin{array}{l}\mathrm{CH}_{3} \\
\mathrm{CO}_{2} \\
\mathrm{CH}_{3}+\mathrm{CO}_{2}\end{array}$ \\
\hline & $p$-Hydroxybenzoic acid & 4.05 & 137.0239 & 137.0261 & $1.5 \mathrm{E} 4$ & $137 \rightarrow 93$ & $\mathrm{CO}_{2}$ \\
\hline \multirow[t]{5}{*}{ A } & Guaiacol & 0.50 & 123.0446 & 123.0442 & $1.4 \mathrm{E} 6$ & $123 \rightarrow 108$ & $\mathrm{CH}_{3}$ \\
\hline & Vanillin & 1.26 & 151.0395 & 151.0391 & $3.0 \mathrm{E} 5$ & $\begin{aligned} 151 \rightarrow 136 \\
151 \rightarrow 108\end{aligned}$ & $\begin{array}{l}\mathrm{CH}_{3} \\
\mathrm{CH}_{3}+\mathrm{CO}\end{array}$ \\
\hline & Acetovanillone & 1.26 & 165.0552 & 165.0553 & $1.7 \mathrm{E} 6$ & $\begin{array}{l}165 \rightarrow 150 \\
165 \rightarrow 122 \\
165 \rightarrow 108\end{array}$ & $\begin{array}{l}\mathrm{CH}_{3} \\
\mathrm{CH}_{3}+\mathrm{CO} \\
\mathrm{CH}_{3}+\mathrm{COCH}_{2}\end{array}$ \\
\hline & $p$-Hydroxybenzaldehyde & 2.49 & 121.0290 & 121.0290 & $1.2 \mathrm{E} 5$ & $121 \rightarrow 92$ & $\mathrm{CHO}$ \\
\hline & $p$-Hydroxyacetophenone & 2.53 & 135.0446 & 135.0441 & $8.2 \mathrm{E} 5$ & $\begin{array}{l}135 \rightarrow 120 \\
135 \rightarrow 108\end{array}$ & $\begin{array}{l}\mathrm{CH}_{3} \\
\mathrm{C}_{2} \mathrm{H}_{3}\end{array}$ \\
\hline \multirow[t]{7}{*}{$\mathrm{X}$} & Guaiacol & 0.51 & 123.0446 & 123.0446 & $4.3 \mathrm{E} 5$ & $123 \rightarrow 108$ & $\mathrm{CH}_{3}$ \\
\hline & Vanillin & 1.27 & 151.0395 & 151.0397 & $5.1 \mathrm{E} 5$ & $\begin{array}{l}151 \rightarrow 136 \\
151 \rightarrow 108\end{array}$ & $\begin{array}{l}\mathrm{CH}_{3} \\
\mathrm{CH}_{3}+\mathrm{CO}\end{array}$ \\
\hline & Acetovanillone & 1.27 & 165.0552 & 165.0556 & $1.1 \mathrm{E} 6$ & $\begin{array}{l}165 \rightarrow 150 \\
165 \rightarrow 122 \\
165 \rightarrow 108\end{array}$ & $\begin{array}{l}\mathrm{CH}_{3} \\
\mathrm{CH}_{3}+\mathrm{CO} \\
\mathrm{CH}_{3}+\mathrm{COCH}_{2}\end{array}$ \\
\hline & $p$-Hydroxybenzaldehyde & 2.51 & 121.0290 & 121.0293 & $3.3 \mathrm{E} 5$ & $121 \rightarrow 92$ & $\mathrm{CHO}$ \\
\hline & $p$-Hydroxyacetophenone & 2.55 & 135.0446 & 135.0445 & $4.8 \mathrm{E} 5$ & $\begin{array}{l}135 \rightarrow 120 \\
135 \rightarrow 108\end{array}$ & $\begin{array}{l}\mathrm{CH}_{3} \\
\mathrm{C}_{2} \mathrm{H}_{3}\end{array}$ \\
\hline & Vanillic acid & 3.29 & 167.0345 & 167.0356 & $2.2 \mathrm{E} 6$ & $\begin{array}{l}167 \rightarrow 152 \\
167 \rightarrow 123 \\
167 \rightarrow 108\end{array}$ & $\begin{array}{l}\mathrm{CH}_{3} \\
\mathrm{CO}_{2} \\
\mathrm{CH}_{3}+\mathrm{CO}_{2}\end{array}$ \\
\hline & $p$-Hydroxybenzoic acid & 4.05 & 137.0239 & 137.0251 & $2.5 \mathrm{E} 5$ & $137 \rightarrow 93$ & $\mathrm{CO}_{2}$ \\
\hline
\end{tabular}


refrigerator, while on the other end, some changes at room temperature were exhibited. The results here suggest that it is better/ recommended to store the depolymerised lignin samples at lower temperatures for enhanced stability behaviour.

\subsection{Supercritical fluid chromatography-mass spectrometry}

The lignin starting material as well as the monomeric-rich fraction extracted from the depolymerised lignin products were qualitatively characterised using the newly introduced SFCMS technique. Two samples from the depolymerised lignin streams produced where chosen beside the Indulin AT sample, residue $A$ which entails the recommended/optimum operating conditions among all the runs performed $\left(240{ }^{\circ} \mathrm{C} ; 2 \mathrm{~min}\right)$ and the run performed at $230^{\circ} \mathrm{C}$ and $1 \mathrm{~min}$ for the sake of drawing a comparative discussion. Figure 10 presents the typical base peak intensity chromatograms for the three investigated samples, and Table 3 reports the most abundant chemical compounds identified along with the MS/MS fragmentation data.

Seven major compounds were identified in the tested samples/fractions: guaiacol, vanillin, acetovanillone, $p$ hydroxyacetophenone, $p$-hydroxybenzaldehyde, vanillic acid and $p$-hydroxybenzoic acid, where the latter three compounds appeared in the starting Indulin kraft lignin sample. It was revealed from the analysis that guaiacol and vanillin are the main products generated from the current depolymerisation approach with higher concentrations/intensities for sample A over sample $\mathrm{X}$, confirming/supporting the results acquired from the SEC attempts.

For the interesting sample A, it can be noted that the compounds exhibiting a retention time higher than 3 min were not identified due to the reason of being at low concentrations. These compounds may comprise vanillic acid and other distinct mono-aromatic moieties with aldehyde, acidic and methoxy functionalities. The decrease/disappearance of these particular compounds in the depolymerised lignin product obtained is due to the increasing severity of the reaction. This finding agrees well with results reported by Beauchet and coworkers [17]. In addition, the high selectivity towards guaiacol formation was attained for the higher reaction time ( $2 \mathrm{~min})$. It can be suggested that the increase in the residence/reaction time (by lowering the feed pump flowrate) assisted in surging the guaiacol production which may have been generated not only from lignin itself but also from the extra depolymerisation of intermediate oligomers. The increased reaction severity also helped - to a great extent - in cleaving the acidic and aldehyde functions which were originally present in the initial starting lignin material. The depolymerised lignin products generated in the present study, i.e. guaiacol and vanillin, are known to have the potential of being key renewable aromatic building blocks for sustainable chemical industry $[4,33]$.

\subsection{Nuclear magnetic resonance spectroscopy}

The integrated ${ }^{1} \mathrm{H}$ NMR spectra obtained for Indulin Kraft starting material and depolymerised lignin samples are shown in Fig. 11. As can be seen, the increased intensities of proton chemical shifts (sharper peaks) of depolymerised lignin sample in comparison to the original lignin material suggest an increase in the degree of depolymerisation and higher formation of low-molecular-weight products.

The measurements on the produced depolymerised residue $A$ show that the aliphatic compounds are rather present, but in very low concentrations. However, the main ${ }^{1} \mathrm{H}$ NMR peaks were assigned to typical aromatic protons (signals 6.0 $8.0 \mathrm{ppm}$ ), besides the methoxyl and aldehyde groups, confirming the presence of small molecular entities within the depolymerised sample and supporting the findings of both SEC and SFC-MS.

It can be interpreted as well from the spectra that the methoxyl content, in particular, was dramatically decreased after the hydrothermal reaction, and this phenomenon agrees well with the reported literature on the hydrothermal treatment of lignin [34]. In addition, the large concentrations of

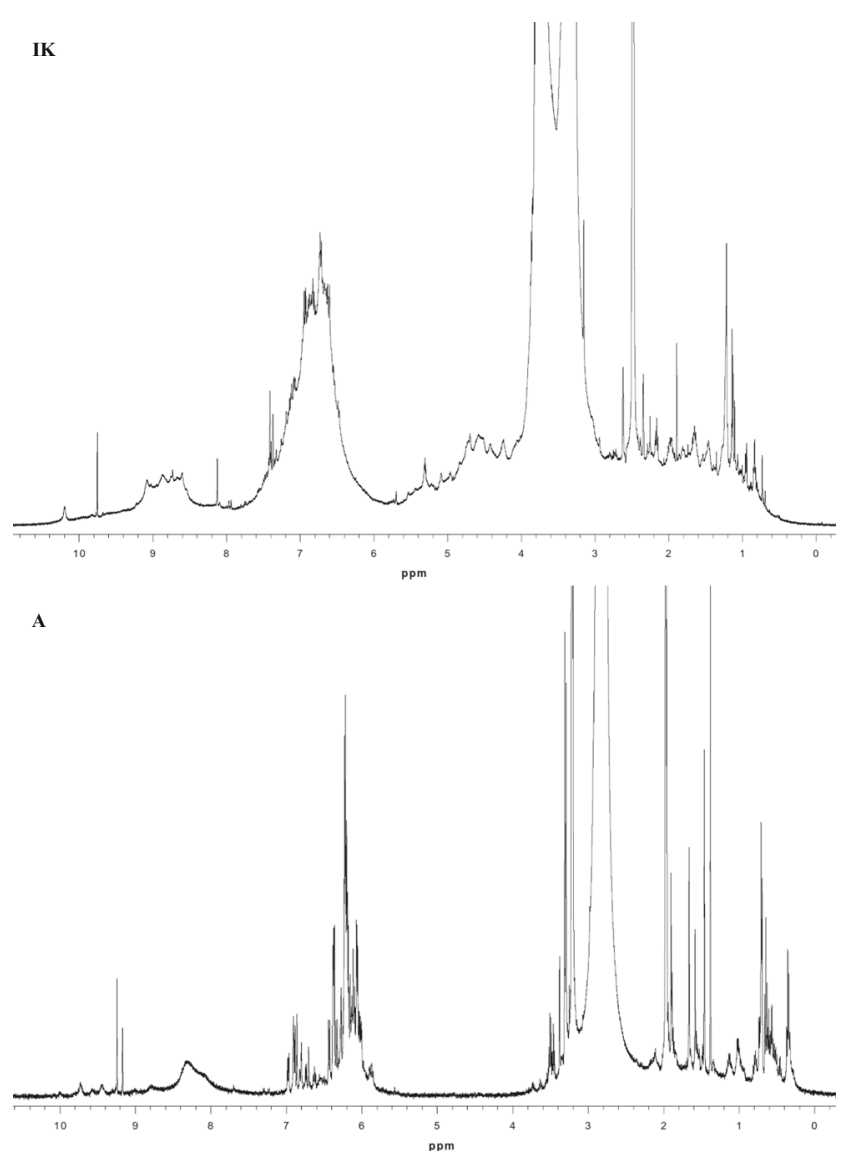

Fig. 11 Solid-state ${ }^{1} \mathrm{H}$ NMR spectra of Indulin AT lignin (IK) and depolymerised lignin residue extracted from the run performed at $T=$ $240{ }^{\circ} \mathrm{C}$ and $\tau=2 \min (\mathrm{A})$ 
hydroxyl and phenolic groups/protons in residue $A$ can explain the increased hydrophilicity of produced aromatic compounds.

2D NMR spectroscopy was run on the depolymerised lignin residue $A$ extracted from the run performed at $T=240{ }^{\circ} \mathrm{C}$ and $\tau=2$ min which is considered to be optimum and compared with the untreated Indulin Kraft lignin sample. The measured spectra were interpreted qualitatively and divided into an aliphatic region $\left(\delta_{\mathrm{C}} / \delta_{\mathrm{H}} 8.0-52.5 / 0.5-4.5 \mathrm{ppm}\right)$, an inter-unit/oxygenated aliphatic region $\left(\delta_{\mathrm{C}} / \delta_{\mathrm{H}} 52.5-90.0 / 2.8\right.$ $5.7 \mathrm{ppm})$ and an aromatic region $\left(\delta_{\mathrm{C}} / \delta_{\mathrm{H}} 105-155 / 6.0\right.$ $9.2 \mathrm{ppm}$ ), as presented in Fig. 12 reporting different chemical shifts acquired. Tentative assignments of ${ }^{13} \mathrm{C}-{ }^{1} \mathrm{H}$ correlation signals/cross-peaks within the 2D NMR spectra were also identified for both lignin samples following recent literature reports $[35,36]$.

In general, guaiacol rings form about $95 \%$ of the major aromatic composition of lignin from softwood origin. Differences in the cross-peak patterns were detected for the two samples in the aliphatic 2D NMR region (Fig. 12; left compartment). The most characteristic cross-peaks of lignin macromolecule are found in the region of $\mathrm{CH}_{2}$ groups (black rings), where they correspond to the primary aliphatic network present in lignin $\mathrm{G}-\mathrm{CH}_{2}-\mathrm{CH}_{2}-\mathrm{CH}(\mathrm{R})-\mathrm{OH} / \mathrm{R}$ located at the $\alpha$ and $\beta$ positions, bounded to the guaiacol ring. Most of the cross-peaks in the depolymerised fractions were found to be connected to an aromatic ring in the $\alpha$-position, which suggests a shortening in the aliphatic chains within the bio-oilresidue $A$-in comparison to the Indulin AT substrate.

The inter-unit aliphatic region (also termed as oxygenated aliphatic region), shown in intermediate compartment of Fig. 12, is the region where carbon atoms bonded to alcohol, ether and Ar-methoxyl groups resonate. The known aliphatic inter-unit linkages $\beta$-O-4 ( $\beta$-ether), $\beta$ - $\beta$ (pinoresinol), $\beta-\beta$ (secoisolariciresinol) and $\beta-5$ (phenylcoumaran) were found for the Indulin AT lignin basic structure. However, the $\beta-1$ (diphenyl ethane), $\alpha-\mathrm{O}-4$ and dibenzodioxocine $(5-5-\alpha, \beta-\mathrm{O}-$ 4) structures were not found/detected, as they were probably hydrolysed during the kraft pulping process. From the visual inspection of the 2D NMR spectra for this region, it can be revealed that all the inter-unit/C-O linkages from Indulin AT lignin have disappeared post pretreatment reaction. This entails that the aliphatic $\mathrm{C}-\mathrm{O}$ bonds have been cleaved under the tested reaction conditions $\left(240{ }^{\circ} \mathrm{C}\right.$ and $2 \mathrm{~min}$ residence time), i.e. in the known lignin inter-unit structures $\beta-O-4, \beta-\beta, \beta-1$ (weak signal) and $\beta-5$. This is indeed an important finding as it subsequently proves that all of the lignin starting material was reacted effectively under basic conditions in our CFR system. It also demonstrates that the amount of oxygen connected to the aliphatic carbons in depolymerised fraction is
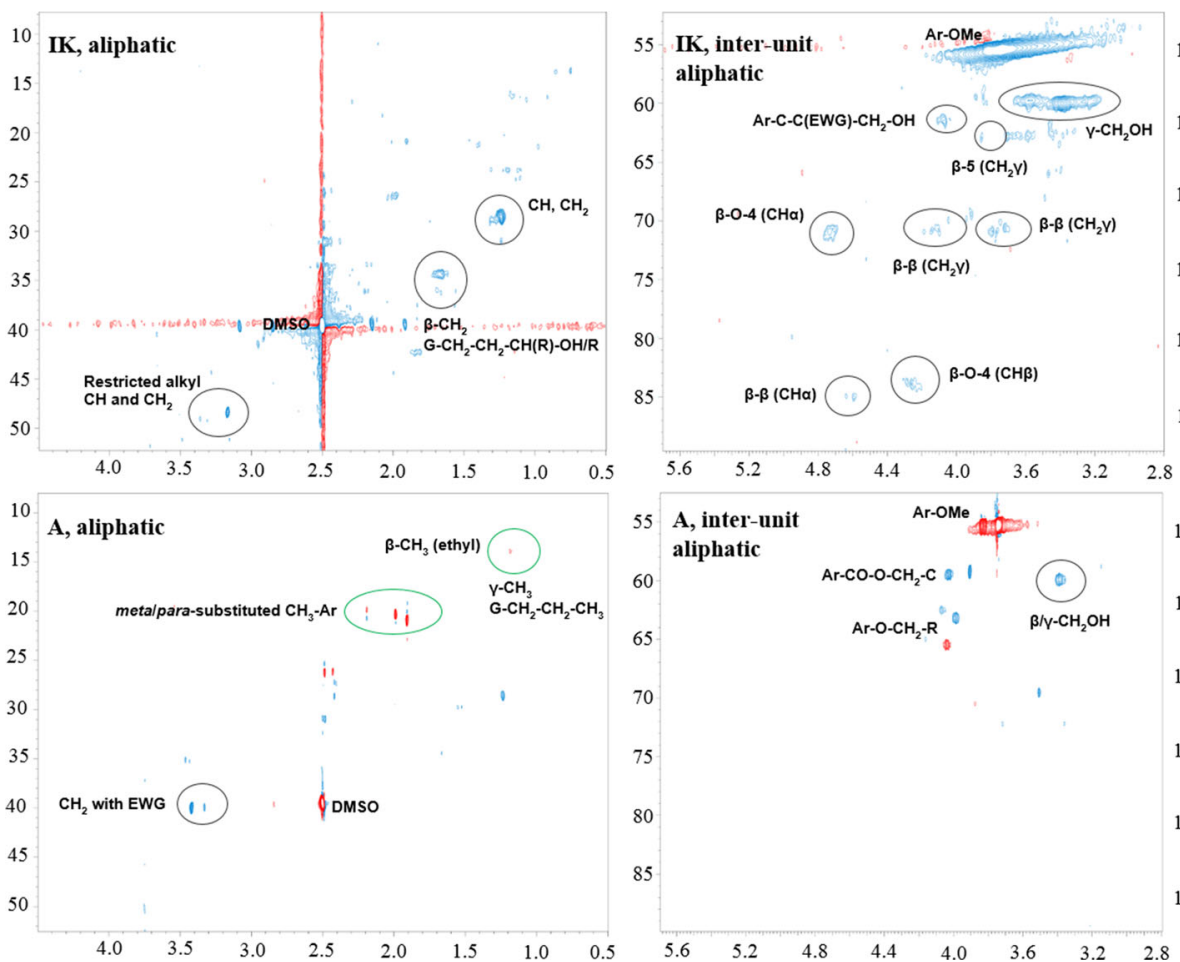
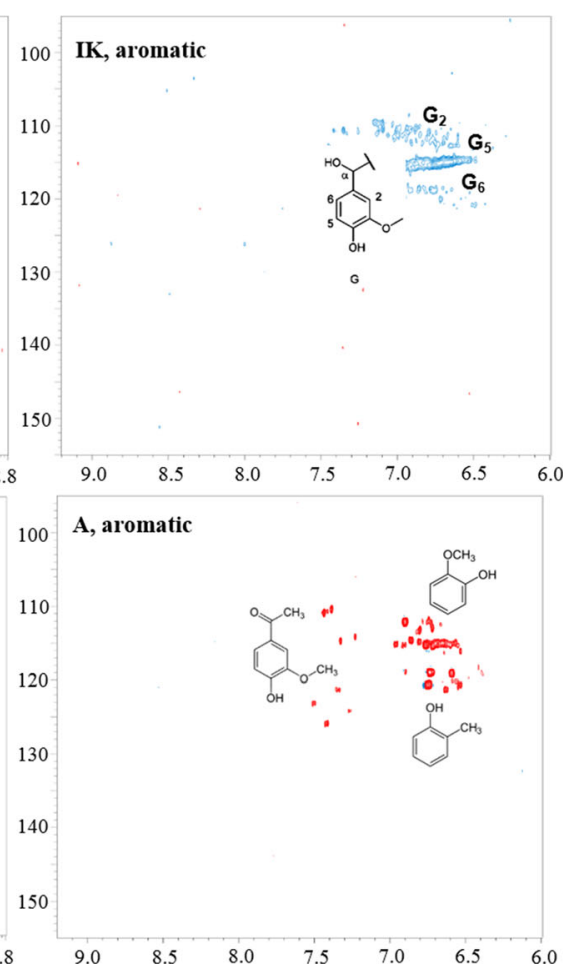

Fig. 12 The aliphatic $\delta_{\mathrm{C}} / \delta_{\mathrm{H}} 8.0-52.5 / 0.5-4.5 \mathrm{ppm}$, inter-unit/oxygenated aliphatic $\delta_{\mathrm{C}} / \delta_{\mathrm{H}} 52.5-90.0 / 2.8-5.7 \mathrm{ppm}$, and aromatic $\delta_{\mathrm{C}} / \delta_{\mathrm{H}} 105-155 /$ 6.0-9.2 ppm regions of 2D NMR spectra obtained for Indulin AT lignin (IK) and depolymerised lignin residue extracted from the run performed at $T=240{ }^{\circ} \mathrm{C}$ and $\tau=2 \mathrm{~min}$ (A). Cross-peaks: $\mathrm{CH}, \mathrm{CH}_{2}$ and $\mathrm{CH}_{3}$ (blue)

for IK sample via normal HSQC and $\mathrm{CH}_{2}$ (blue); $\mathrm{CH} / \mathrm{CH}_{3}$ (red) for sample $A$ via edited HSQC experiments. Black rings correspond to lignin structural motifs, and green rings are new cross-peaks found in depolymerised lignin sample (colour figure online) 
lowered. To note, the content of atomic oxygen comes almost exclusively from oxygen-containing groups $(-\mathrm{OH}$ and $-\mathrm{OMe} /$ $\mathrm{R})$ connected to the aromatic ring, like phenols, guaiacols and other aromatic ethers.

Looking into the aromatic region, right compartment of Fig. 12, it was found that the main $\mathrm{CH}$ cross-peaks/signals of Indulin AT lignin correspond to the guaiacol ring $(\mathrm{G})$ and the recognised substitution pattern of softwood kraft lignin, i.e. $\mathrm{G}_{2}, \mathrm{G}_{5}$ and $\mathrm{G}_{6}$. It can further be seen that depolymerised lignin — residue $A$ - exhibited a pattern of cross-peaks different than that of Indulin AT lignin. One of the principal reasons for this is that all monomeric/dimeric low-molecular-weight aromatics derived/produced ended up in this fraction. It was also confirmed from the SFC-MS analysis that phenol and phenol derivatives, e.g. guaiacol, dominate this residue/fraction, complementing such results.

\section{Conclusions}

Base-catalysed depolymerisation experiments on industrially generated lignin streams have been demonstrated using a newtailored continuous flow reactor system. Kraft lignin was successfully converted into a phenolic bio-oil consisting of monomeric/oligomeric aromatic compounds. The optimum operating conditions for such conversion, yielding the highest concentration of low-molecular-weight aromatics and below the coking point, were achieved at $240{ }^{\circ} \mathrm{C}, 2 \mathrm{~min}, 5 \mathrm{wt} \%$ lignin loading and $\mathrm{NaOH} /$ lignin ratio $\approx 1(w / w)$. The depolymerisation reaction appeared to be a $\mathrm{NaOH}$ consuming process, and this is directly proportional to the increase in reaction temperature and residence time, supported by RI profiles. The aliphatic $\mathrm{C}-\mathrm{O}$ bonds have been cleaved under the tested reaction conditions in the lignin inter-unit structures $(\beta-$ $0-4, \beta-\beta, \beta-1, \beta-5)$, confirming that an effective lignin depolymerisation has been encountered. Controlling the reaction and storage temperatures is crucial for minimising char formation and preventing recondensation/repolymerisation reactions. Monomeric phenolic compounds (guaiacol, vanillin, apocynin, piceol) were identified from the current depolymerisation approach, generating a pool of chemically viable phenolic building blocks that can be considered for further high-value applications within the food and/or pharmaceutical industries. The present lignin depolymerisation reaction also enabled the production of partly deoxygenated dimeric/oligomeric fractions that could be utilised in liquid fuels applications and other renewable energy alternatives.

Acknowledgments Special thanks are due to Göran Carlström for assisting in NMR experiments, Jens Prothmann and Mingzhe Sun for helping in SFC-MS measurements and Johan Thuvander for the technical support with SEC.
Funding information This work was financed by the Swedish Foundation for Strategic Research (SSF) through the grant contract RBP14-0052. Financial supports from the Swedish Energy Agency (contract no. 41288-1), Preem, SunCarbon and Sveaskog are also acknowledged.

Open Access This article is distributed under the terms of the Creative Commons Attribution 4.0 International License (http:// creativecommons.org/licenses/by/4.0/), which permits unrestricted use, distribution, and reproduction in any medium, provided you give appropriate credit to the original author(s) and the source, provide a link to the Creative Commons license, and indicate if changes were made.

\section{References}

1. Ragauskas AJ, Beckham GT, Biddy MJ, Chandra R, Chen F, Davis MF, Davison BH, Dixon RA, Gilna P, Keller M, Langan P, Naskar AK, Saddler JN, Tschaplinski TJ, Tuskan GA, Wyman CE (2014) Lignin valorization: improving lignin processing in the biorefinery. Science 344(6185):1246843. https://doi.org/10.1126/science. 1246843

2. Rinaldi R, Jastrzebski R, Clough MT, Ralph J, Kennema M, Bruijnincx PCA, Weckhuysen BM (2016) Paving the way for lignin valorisation: recent advances in bioengineering, biorefining and catalysis. Angew Chemie Int Ed 55(29):8164-8215. https://doi.org/ 10.1002/anie.201510351

3. Abdelaziz OY, Brink DP, Prothmann J, Ravi K, Sun M, GarcíaHidalgo J, Sandahl M, Hulteberg CP, Turner C, Lidén G, GorwaGrauslund MF (2016) Biological valorization of low molecular weight lignin. Biotechnol Adv 34(8):1318-1346. https://doi.org/ 10.1016/j.biotechadv.2016.10.001

4. Li C, Zhao X, Wang A, Huber GW, Zhang T (2015) Catalytic transformation of lignin for the production of chemicals and fuels. Chem Rev 115(21):11559-11624. https://doi.org/10.1021/acs. chemrev.5b00155

5. Montazeri M, Eckelman MJ (2016) Life cycle assessment of catechols from lignin depolymerization. ACS Sustain Chem Eng 4(3): 708-718. https://doi.org/10.1021/acssuschemeng.5b00550

6. Ayyachamy M, Cliffe FE, Coyne JM, Collier J, Tuohy MG (2013) Lignin: untapped biopolymers in biomass conversion technologies. Biomass Convers Biorefinery 3(3):255-269. https://doi.org/10. 1007/s13399-013-0084-4

7. Sjöström E (1993) Wood chemistry: fundamentals and applications, 2nd edn. Academic Press, Inc., San Diego, California

8. Calvo-Flores FG, Dobado JA, Isac-García J, Martín-Martínez FJ (2015) Lignin and Lignans as renewable raw materials: chemistry, Technology and Applications. John Wiley \& Sons, Ltd, Chichester. https://doi.org/10.1002/9781118682784

9. Yang L, Li Y, Savage PE (2014) Hydrolytic cleavage of C-O linkages in lignin model compounds catalyzed by water-tolerant Lewis acids. Ind Eng Chem Res 53(7):2633-2639. https://doi.org/10. $1021 /$ ie $403545 n$

10. Xu C, Arancon RAD, Labidi J, Luque R (2014) Lignin depolymerisation strategies: towards valuable chemicals and fuels. Chem Soc Rev 43(22):7485-7500. https://doi.org/10.1039/ C4CS00235K

11. Toledano A, Serrano L, Labidi J (2014) Improving base catalyzed lignin depolymerization by avoiding lignin repolymerization. Fuel 116:617-624. https://doi.org/10.1016/j.fuel.2013.08.071

12. Katahira R, Mittal A, McKinney K, Chen X, Tucker MP, Johnson DK, Beckham GT (2016) Base-catalyzed depolymerization of biorefinery lignins. ACS Sustain Chem Eng 4(3):1474-1486. https://doi.org/10.1021/acssuschemeng.5b01451 
13. Miller JE, Evans L, Littlewolf A, Trudell DE (1999) Batch microreactor studies of lignin and lignin model compound depolymerization by bases in alcohol solvents. Fuel 78(11):1363-1366. https://doi.org/10.1016/S0016-2361(99)00072-1

14. Shabtai JS, Zmierczak WW, Chornet E (2001) Process for conversion of lignin to reformulated, partially oxygenated gasoline

15. Dos Santos PSB, Erdocia X, Gatto DA, Labidi J (2016) Bio-oil from base-catalyzed depolymerization of organosolv lignin as an antifungal agent for wood. Wood Sci Technol 50(3):599-615. https://doi.org/10.1007/s00226-015-0795-8

16. Mahmood N, Yuan Z, Schmidt J, Xu C (2013) Production of polyols via direct hydrolysis of kraft lignin: effect of process parameters. Bioresour Technol 139:13-20. https://doi.org/10.1016/j. biortech.2013.03.199

17. Beauchet R, Monteil-Rivera F, Lavoie JM (2012) Conversion of lignin to aromatic-based chemicals (L-chems) and biofuels (Lfuels). Bioresour Technol 121:328-334. https://doi.org/10.1016/j. biortech.2012.06.061

18. Roberts VM, Stein V, Reiner T, Lemonidou A, Li X, Lercher JA (2011) Towards quantitative catalytic lignin depolymerization. Chem - A Eur J 17(21):5939-5948. https://doi.org/10.1002/chem. 201002438

19. Li J, Henriksson G, Gellerstedt G (2007) Lignin depolymerization/ repolymerization and its critical role for delignification of aspen wood by steam explosion. Bioresour Technol 98(16):3061-3068. https://doi.org/10.1016/j.biortech.2006.10.018

20. Gosselink RJA, Teunissen W, van Dam JEG, de Jong E, Gellerstedt G, Scott e, Sanders JPM (2012) Lignin depolymerisation in supercritical carbon dioxide/acetone/water fluid for the production of aromatic chemicals. Bioresour Technol 106:173-177. https://doi. org/10.1016/j.biortech.2011.11.121

21. Rahimi A, Ulbrich A, Coon JJ, Stahl SS (2014) Formic-acidinduced depolymerization of oxidized lignin to aromatics. Nature 515(7526):249-252. https://doi.org/10.1038/nature13867

22. Huang S, Mahmood N, Tymchyshyn M, Yuan Z, Xu CC (2014) Reductive de-polymerization of kraft lignin for chemicals and fuels using formic acid as an in-situ hydrogen source. Bioresour Technol 171:95-102. https://doi.org/10.1016/j.biortech.2014.08.045

23. Huang X, Korányi TI, Boot MD, Hensen EJM (2014) Catalytic depolymerization of lignin in supercritical ethanol. ChemSusChem 7(8):2276-2288. https://doi.org/10.1002/cssc.201402094

24. Galkin MV, Samec JSM (2016) Lignin valorization through catalytic lignocellulose fractionation: a fundamental platform for the future biorefinery. ChemSusChem 9(13):1544-1558. https://doi. org/10.1002/cssc.201600237

25. Zakzeski J, Bruijnincx PCA, Jongerius AL, Weckhuysen BM (2010) The catalytic valorization of lignin for the production of renewable chemicals. Chem Rev 110(6):3552-3599. https://doi. org/10.1021/cr900354u
26. Hu Z, Du X, Liu J, Chang H, Jameel H (2016) Structural characterization of pine kraft lignin: BioChoice lignin vs Indulin AT. J. Wood Chem. Technol. 36(6):432-446. https://doi.org/10.1080/ 02773813.2016 .1214732

27. Sheng C, Azevedo JLT (2005) Estimating the higher heating value of biomass fuels from basic analysis data. Biomass Bioenergy 28(5):499-507. https://doi.org/10.1016/j.biombioe.2004.11.008

28. Abdelaziz OY, Hulteberg CP (2017) Physicochemical characterisation of technical lignins for their potential valorisation. Waste Biomass Valoriz. 8(3):859-869. https://doi.org/10.1007/s12649016-9643-9

29. Prothmann J, Sun M, Spégel P, Sandahl M, Turner C (2017) Ultrahigh-performance supercritical fluid chromatography with quadrupole-time-of-flight mass spectrometry (UHPSFC/QTOFMS) for analysis of lignin-derived monomeric compounds in processed lignin samples. Anal Bioanal Chem. https://doi.org/10.1007/ s00216-017-0663-5

30. Brittain AD, Chrisandina NJ, Cooper RE, Buchanan M, Cort JR, Olarte MV, Sievers C (2017) Quenching of reactive intermediates during mechanochemical depolymerization of lignin. Catal Today. https://doi.org/10.1016/j.cattod.2017.04.066

31. Nguyen Lyckeskog H, Mattsson C, Åmand L-E, Olausson L, Andersson SI, Vamling L, Theliander H (2016) Storage stability of bio-oils derived from the catalytic conversion of softwood kraft lignin in subcritical water. Energy Fuel 30(4):3097-3106. https:// doi.org/10.1021/acs.energyfuels.6b00087

32. Saisu M, Sato T, Watanabe M, Adschiri T, Arai K (2003) Conversion of lignin with supercritical water-phenol mixtures. Energy Fuel 17(4):922-928. https://doi.org/10.1021/ef0202844

33. Fache M, Boutevin B, Caillol S (2016) Vanillin production from lignin and its use as a renewable chemical. ACS Sustain Chem Eng 4(1):35-46. https://doi.org/10.1021/acssuschemeng.5b01344

34. Matsushita $\mathrm{Y}$, Jo E-K, Inakoshi R, Yagami S, Takamoto N, Fukushima K, Lee SC (2013) Hydrothermal reaction of sulfuric acid lignin generated as a by-product during bioethanol production using lignocellulosic materials to convert bioactive agents. Ind Crop Prod 42:181-188. https://doi.org/10.1016/j. indcrop.2012.05.030

35. Mattsson C, Andersson S-I, Belkheiri T, Le Å, Olausson L, Vamling L, Theliander H (2016) Using 2D NMR to characterize the structure of the low and high molecular weight fractions of biooil obtained from LignoBoost ${ }^{\mathrm{TM}} \mathrm{kraft}$ lignin depolymerized in subcritical water. Biomass Bioenergy 95:364-377. https://doi.org/10. 1016/j.biombioe.2016.09.004

36. Constant S, Wienk HLJ, Frissen AE, Peinder P, Boelens R, van Es DS, Grisel RJH, Weckhuysen BM, Huijgen WJJ, Gosselink RJA, Bruijnincx PCA (2016) New insights into the structure and composition of technical lignins: a comparative characterisation study. Green Chem 18(9):2651-2665. https://doi.org/10.1039/ C5GC03043A 